approximate formula for the 3 - $\mathrm{dB}$ cutoff frequency as a function of polynomial order $N$ and impulse response halflength $M$. Engineers with a frequencydomain mindset (like the author) may find this useful if they choose to use $\mathrm{S}-\mathrm{G}$ filters in their application.

\section{AUTHOR}

RonaldW. Schafer (ron.schafer@hp.com) is an HP Fellow in the Mobile and Immersive Experience Lab at HP Labs, Palo Alto, California, where he is involved in research on acoustic and audio signal processing. From 1974 to 2004, he was John and Marilu McCarty Professor of the School of Electrical and Computer Engineering at Georgia Tech. He is the coauthor of several DSP textbooks including Discrete-Time Signal Processing (with Oppenheim), Signal Processing First (with McClellan and Yoder), and Theory and Applications of Digital Speech Processing (with Rabiner).

\section{REFERENCES}

[1] K. Pandia, S. Revindran, R. Cole, G. Kovacs, and L. Giaovangrandi, "Motion artifact cancellation to obtain heart sounds from a single chestworn accelerometer," in Proc. ICASSP-2010, 2010 pp. 590-593.

[2] R. W. Schafer, "On the frequency-domain properties of Savitzky-Golay filter," in Proc. 2011 DSP/SPE Workshop, Sedona, AZ, Jan. 2011, pp. 54-59.

[3] A. Savitzky and M. J. E. Golay, "Soothing and differentiation of data by simplified least squares procedures," Anal. Chem., vol. 36, pp. 1627-1639, 1964.

[4] J. Riordon, E. Zubritsky, and A. Newman, "Top 10 articles," Anal. Chem., vol. 72, no. 9, pp. 324A329A, May 2000.
[5] M. Sühling, M. Arigovindan, P. Hunziker, and M. Unser, "Multiresolution moment filters: Theory and applications," IEEE Trans. Image Processing, vol. 13, no. 4, pp. 484-495, Apr. 2004

[6] M. U. A. Bromba and H. Ziegler, "Application hints for Savitzky-Golay smoothing filters," Anal. Chem., vol. 53, no. 11, pp. 1583-1586, Sept. 1981.

[7] R. W. Hamming, Digital Filters, 3rd ed. Englewood Cliffs, NJ: Prentice-Hall, 1989.

[8] S. J. Orfanidis. (1995-2009). Introduction to signal processing [Online]. Available: www.ece. rutgers.edu/ orfanidis/intro2sp

[9] P.-O. Persson and G. Strang, "Smoothing by Savitzky-Golay and Legendre filters," IMA Vol. Math. Systems Theory Biol., Comm., Comp., and Finance, vol. 134, pp. 301-316, 2003.

[10] W. H. Press, S. A. Teukolsky, W. T. Vertterling, and B. P. Flannery, Numerical Recipes, 3rd ed. Cambridge, U. K.: Cambridge Univ. Press, 2007.

[11] A. V. Oppenheim and R. W. Schafer, DiscreteTime Signal Processing. 3rd ed. Upper Saddle River, NJ: Pearson, 2010

\title{
Low-Pass Filtering of Irregularly Sampled Signals Using a Set Theoretic Framework
}

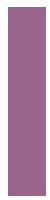

$\mathrm{n}$ this article, the goal is to show that it is possible to filter nonuniformly sampled signals according to specs defined in the Fourier domain. In many practical applications, it is necessary to filter irregularly sampled data including seismic signal processing, synthetic aperture radar (SAR) imaging systems, three-dimensional (3-D) meshes, and digital terrain models [1], [2]. In almost all of these practical problems, it is possible to define the desired filtering solution in a set theoretic framework. This lecture note presents a new method for filtering irregularly sampled data by defining stopband tolerance regions in the Fourier domain and time-domain upper and lower bounds on the signal samples as a part of the filtering process. Since there are specifications in both time and frequency domains, it is possible to iterate between time and frequency domains using the fast Fourier transform (FFT) while imposing the constraints in each domain.

\section{RELEVANCE}

The ideas presented here can be used to develop filtering algorithms for irregularly sampled one or higher dimensional data. It can be used as a teaching material in advanced undergraduate and graduate discrete-time signal processing, optimization as well as applied mathematics courses.

\section{PREREQUISITES}

The prerequisites for understanding this article's material are linear algebra, discrete-time signal processing, and basic optimization theory.

\section{PROBLEM STATEMENT}

Let us assume that samples $x_{c}\left(t_{i}\right)$, $i=0,1,2, \ldots, L-1$, of a continuous time-domain signal $x_{c}(t)$ are available. These samples may not be on an uniform sampling grid. Let us define $x_{d}[n]=x_{c}\left(n T_{s}\right)$ as the uniformly sampled version of this signal. We assume that the sampling period $T_{s}$ is sufficiently small (below the Nyquist period) for the signal $x_{c}(t)$. In a typical discretetime filtering problem, we have $x_{d}[n]$ or its noisy version, and we apply a discrete-time low-pass filter to the uniformly sampled signal $x_{d}[n]$. However, $x_{d}[n]$ is not available in this problem. Only nonuniformly sampled data $x_{c}\left(t_{i}\right)$, $i=0,1,2, \ldots, L-1$ are available in this problem.

\section{GOAL}

Our goal is to low-pass filter the nonuniformly sampled data $x_{c}\left(t_{i}\right)$ according 


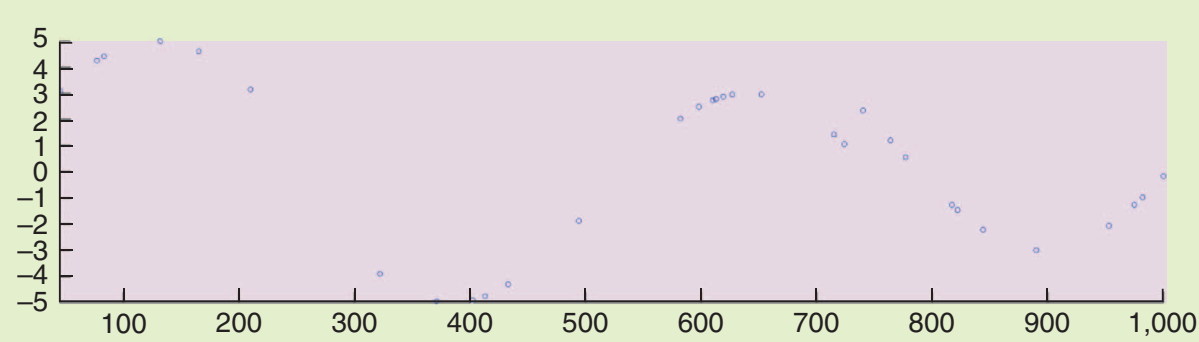

(a)

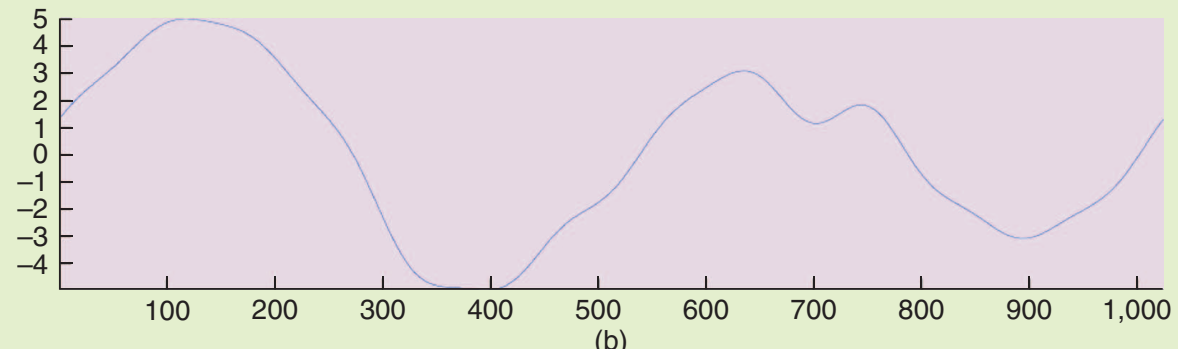

[FIG1] (a) 32-point nonuniform sampled version of the Heavisine function. (b) The 1,024-point interpolated versions of the function given at (a).

to a given cutoff frequency. One can try to interpolate available samples to the regular grid and apply a discrete-time filter to the data but this will amplify the noise because the available samples may be corrupted by noise [3]. In fact, only noisy samples are available in some problems [4].

\section{PROPOSED SOLUTION}

The proposed filtering algorithm is essentially a variant of the well-known Papoulis-Gerchberg interpolation method [1], [5]-[10] and our earlier finite impulse response (FIR) filter design method [11]. The solution is based on the projections onto convex sets (POCSs) framework. In this approach, specifications in the time and frequency domain are formulated as sets and a signal in the intersection of constraint sets is defined as the solution, which can be obtained in an iterative manner. In each iteration, the FFT algorithm is used to go back and forth between the time and frequency domains.

In many signal reconstruction and band-limited interpolation problems [1], [5]-[7], Fourier domain information is represented using a set, which is defined as follows:

$C_{p}=\left\{x: X\left(e^{j w}\right)=0\right.$ for $\left.w_{c} \leq w \leq \pi\right\}$,

IN MANY SIGNAL
RECONSTRUCTION
AND BAND-LIMITED
INTERPOLATION PROBLEMS,
FOURIER DOMAIN
INFORMATION
IS REPRESENTED
USING A SET.

where $X\left(e^{j w}\right)$ is the discrete-time Fourier transform (DTFT) of the discrete-time signal $x[n]$ and $w_{c}$ is the band-limitedness condition or the desired normalized angular low-pass cutoff frequency [1], [5], [6]. This condition is imposed on a given signal $x_{o}[n]$ by orthogonal projection onto the set $C_{p}$ as follows. The projection $x_{p}$ is obtained by simply imposing the frequency domain constraint on the signals

$X_{p}\left(e^{j w}\right)=\left\{\begin{array}{ll}X_{o}\left(e^{j w}\right) & \text { for } 0 \leq w \leq w_{c} \\ 0 & \text { for } w>w_{c}\end{array}\right.$,

where $X_{o}\left(e^{j w}\right)$ and $X_{p}\left(e^{j w}\right)$ are the DTFTs of $x_{o}$ and $x_{p}$, respectively. Members of the set $C_{p}$ are infinite extent signals, so the FFT size should be large during the implementation of the projection on to the set $C_{p}$.
This approach is different from the Papoulis-Gerchberg type method [1], [5], [6] because it allows the signal to have some high-frequency components according to the tolerance parameter $\delta_{s}$. The use of the stopband and the transition regions eliminates ringing artifacts due to the Gibbs phenomenon. We define another set corresponding to the stopband condition in the Fourier domain as follows:

$C_{s}=\left\{x:\left|X\left(e^{j w}\right)\right| \leq \delta_{s}\right.$ for $\left.w_{s} \leq w \leq \pi\right\}$,

where the stopband frequency $w_{s}>w_{c}$. The set $C_{s}$ is also a convex set [6], [12], and we can impose this condition on iterates during iterative filtering. We find a member $x_{g}$ of the set $C_{s}$ corresponding to a given signal $x_{o}[n]$ as follows:

$$
\begin{aligned}
& X_{g}\left(e^{j w}\right)= \\
& \begin{cases}X_{o}\left(e^{j w}\right) & \text { for } 0<w<w_{s} \\
X_{o}\left(e^{j w}\right) & \text { for }\left|X_{o}\left(e^{j w}\right)\right| \leq \delta_{s}, w \geq w_{s}, \\
\delta_{s} e^{j \phi_{o}(w)} & \text { for }\left|X_{o}\left(e^{j w}\right)\right| \geq \delta_{s}, w \geq w_{s}\end{cases}
\end{aligned}
$$

where $\phi_{o}(w)$ is the phase of $X_{o}\left(e^{j w}\right)$. Clearly, $x_{g}$ is in the set $C_{s}$. In our implementation, the set $C_{s}$ plays the key role rather than the set $C_{p}$ because almost all signals that we encounter in 


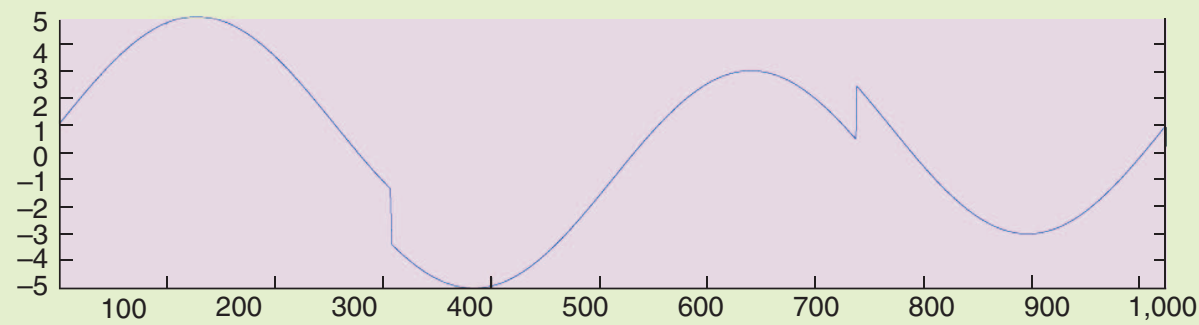

(a)

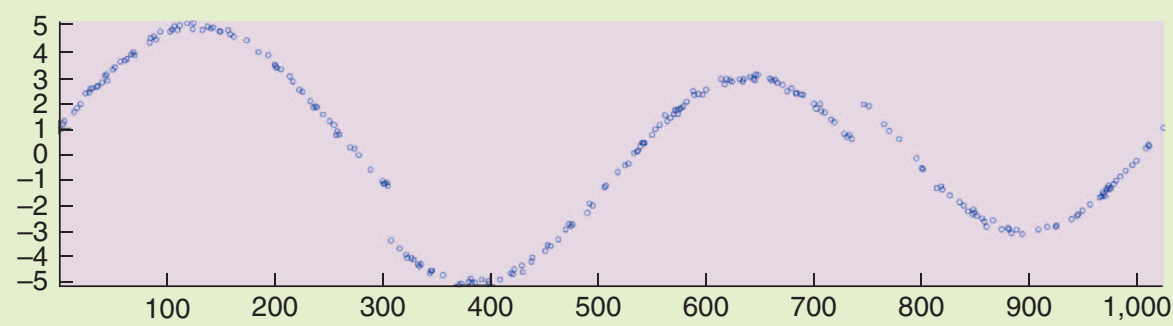

(b)

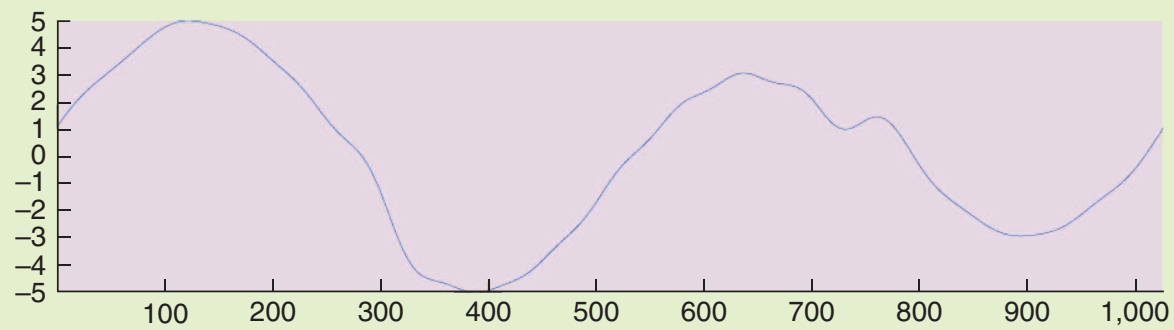

(c)

[FIG2] (a) The Heavisine signal, (b) 256 Heavisine signal samples corrupted by additive Gaussian noise with variance $\sigma=0.3$, and (c) 1,024 point restored signal using the samples given in (b).

practice are not band-limited signals. Most signals have some high-frequency content. The frequency band $\left(w_{c}, w_{s}\right)$ corresponds to the transition band used in ordinary discrete-time filter design.

As pointed out above, we use a sampling period, which is smaller than the Nyquist period. Let us assume that $0, T_{s}, 2 T_{s}, \ldots,(N-1) T_{s}$ is a dense grid covering $t_{i}, i=0,1,2, \ldots, L-1$ and let us also assume that all $t_{i}<t_{i+1}$ and $t_{i} \geq 0$ and $t_{L-1} \leq(N-1) T_{s}$ without loss of generality. The set describing the time-domain information is defined using the regular sampling grid $0, T_{s}, 2 T_{s}, \ldots,(N-1) T_{s}$. Let us assume that the sample at $t=t_{i}$ is close to $n T_{s}$. We impose upper and lower bounds on $x[n]$ as follows:

$$
x_{c}\left(t_{i}\right)-e_{i} \leq x[n] \leq x_{c}\left(t_{i}\right)+e_{i}
$$

and the corresponding time-domain set is defined as

$$
C_{i}=\left\{x: x_{c}\left(t_{i}\right)-e_{i} \leq x[n] \leq x_{c}\left(t_{i}\right)+e_{i}\right\},
$$

where the time-domain bound parameter $e_{i}$ can be either selected as a constant value or as an $\alpha$-percent of $x_{c}\left(t_{i}\right)$ in a

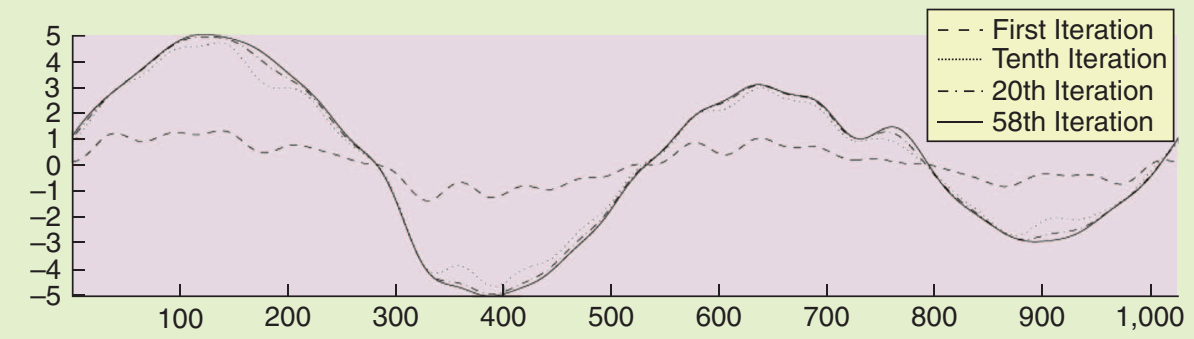

[FIG3] Restored signals after 1, 10, 20, and 58 iteration rounds. 


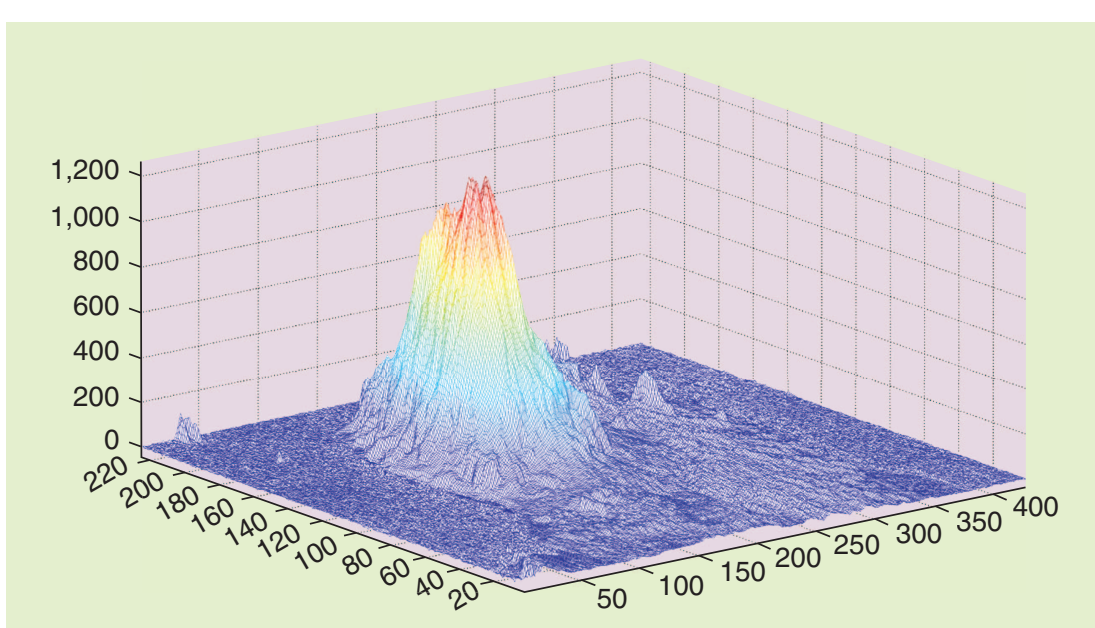

[FIG4] The original terrain model. The original model consists of $225 \times 425$ samples.

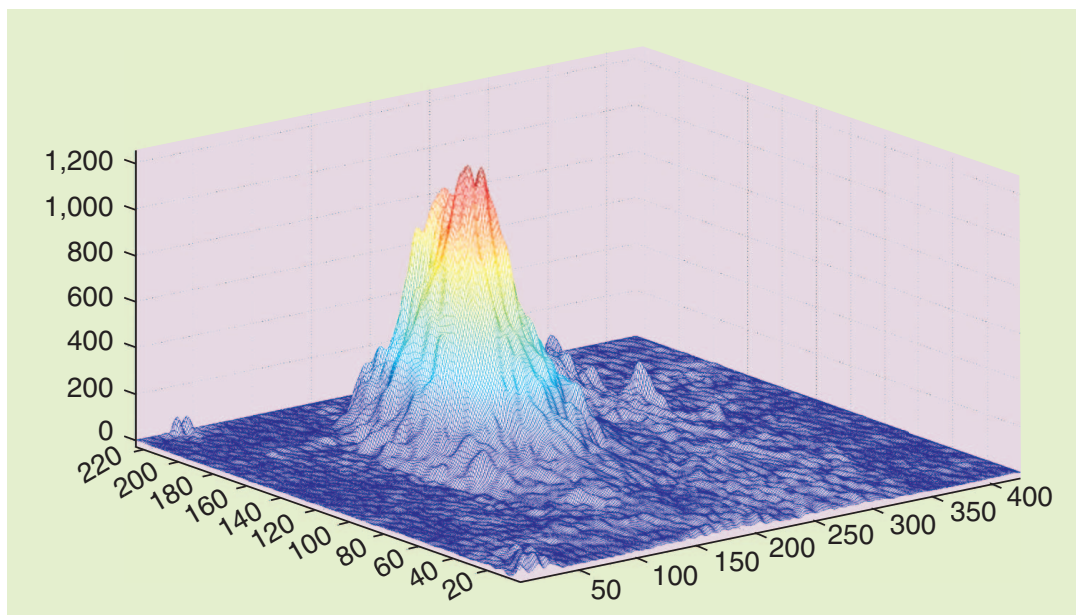

[FIG5] Reconstructed model using one fourth of the randomly chosen samples of the original model. The reconstruction parameters are $w_{c}=\pi / 4, \delta_{s}=0.03$, and $e_{i}=0.01$.

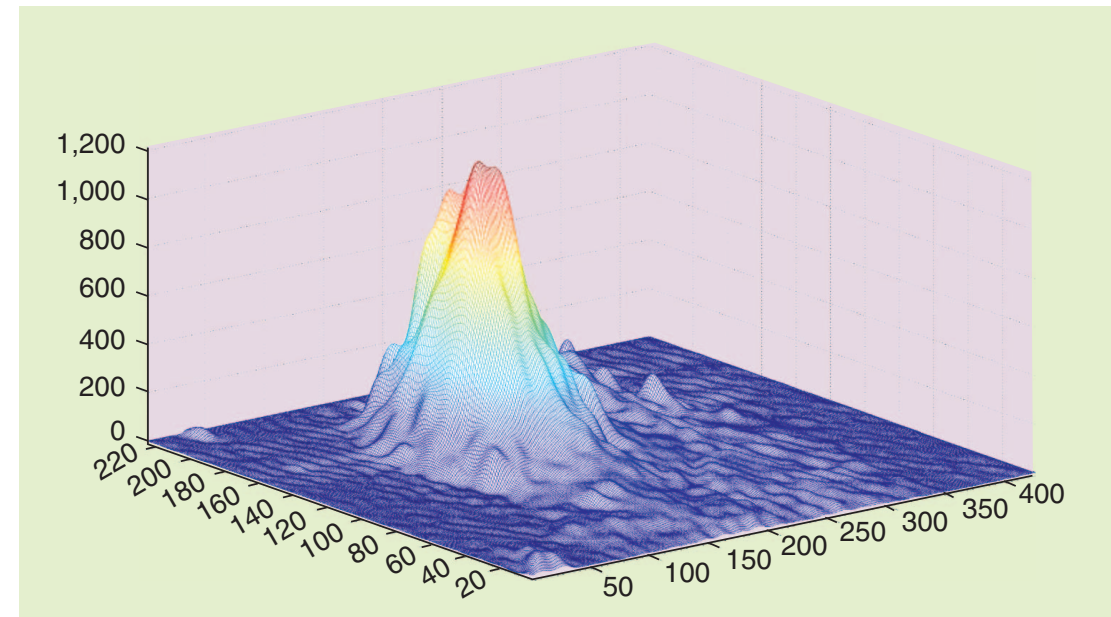

[FIG6] Reconstructed model using one eighth of the randomly chosen samples of the original model. The reconstruction parameters are $w_{c}=\pi / 8, \delta_{s}=0.03$, and $e_{i}=0.01$. practical implementation. Although we do not know the signal value at $n T_{s}$ on the regular grid, it should be close to the sample value $x_{c}\left(t_{i}\right)$ due to the low-pass nature of the desired signal. Therefore, we model this information by imposing upper and lower bounds on the discretetime signal in sets $C_{i}, i=0,1,2$, $\ldots, L-1$. Furthermore, samples may be corrupted by noise and upper and lower bounds on sample values provide robustness against noise. If there are two signal samples close to $x[n]$, the grid size can be increased, i.e., the sampling period can be reduced so that there is one $x[n]$ corresponding to each $x_{c}\left(t_{i}\right)$. Other time-domain constraints that can be used in an iterative algorithm include the positivity constraint $x[n] \geq 0$, if the signal is nonnegative, and the finite energy set

$$
C_{E}=\left\{x:\|x\|^{2} \leq E\right\},
$$

which is introduced in [6] for band-limited interpolation problems to provide robustness against noise.

\section{ITERATIVE FILTERING ALGORITHM}

The iterative filtering algorithm consists of going back and forth between time and frequency domains and imposing the time and frequency constraints on iterates. We start with an arbitrary initial signal $x_{o}[n]$. We project it onto sets $C_{i}$ by using the time-domain constraints defined in (5) and obtain the first iterate $x_{1}[n]$. Next, we need to compute the DTFT of $x_{1}[n]$ and impose the frequency domain constraint defined in (4) to obtain $X_{2}$.

We then compute the inverse-DTFT of $X_{2}$ to obtain $x_{2}$. At this stage, other time-domain constraints such as positivity and finite energy can be also imposed on $x_{2}$, if the signal is known to be a nonnegative signal. Once $x_{2}$ is obtained, it probably violates the time-domain constraints defined by inequalities (5). Therefore, $x_{3}$ is obtained by imposing the constraints on $x_{2}$. The iterates defined in this manner converge to a signal in the intersection of the timedomain set $C_{i}$ and the frequency domain set $C_{s}$, if they intersect. In other words, 
we eventually find a low-pass filtered version of the signal $x_{c}(t)$ on the regular grid defined by $0, T_{s}, 2 T_{s}, \ldots,(N-1) T_{s}$. If the intersection of the sets $C_{i}$ and $C_{s}$ is empty then either the bounds $e_{i}$ should be increased or the cutoff frequency $w_{s}$ should be increased.

The iterative algorithm is globally convergent regardless of the initial starting signal, $x_{o}[n]$. The proof of convergence is due to the POCSs theorem [6], [7], because the sets $C_{s}, C_{i}$, and $C_{E}$ are all convex sets in $l_{2}$. Successive orthogonal projections onto these sets lead to a solution, which is in the intersection of $C_{s}$, $C_{i}$, and $C_{E}$. Papoulis-Gerchberg type iterations jumping back and forth between time and frequency domains converge in a relatively slow manner. Convergence speed can be increased using the nonorthogonal projection methods described in [6], [7], and [13].

\section{NUMERICAL EXAMPLES}

Figure 1 demonstrates the use of the low-pass filtering method for Donoho's Heavisine signal shown in Figure 2(a). Due to the edges, this signal has highfrequency content. Therefore neither the strict band-limited interpolation employing the set $C_{p}$ nor the use of spline interpolation will produce satisfactory results for this signal as demonstrated in [3].

In Figure 1, the number of available samples of the Heavisine signal is 32 . Available samples are shown in Figure 1(a). In this case, the cutoff frequency $w=(2 \cdot \pi \cdot 11) / 1024$, stopband parameter, $\delta_{s}=0.03$, and the time domain bound parameter $e_{i}=0.01$. The restored signal is shown in Figure $2(\mathrm{~b})$. The restored signal is similar to the signal obtained using the wavelet domain methods described in [3].

In Figure 2, we randomly chose 256 points from the Heavisine signal, and we estimate the underlying continuous-time signal at 1,024 uniformly selected instances, i.e., $x[n], n=0,1,2, \ldots, 1023$. The available signal samples are corrupted by Gaussian noise with variance $\sigma=0.3$ as in [3]. Corrupted Heavisine signal samples are shown in Figure 2(b). The restored signal using the sets $C_{s}$ and $C_{i}$ is shown in Figure 2(c). The cutoff frequency

\section{THE ITERATIVE FILTERING ALGORITHM CONSISTS OF GOING BACK AND FORTH BETWEEN TIME AND FREQUENCY DOMAINS AND IMPOSING THE TIME AND FREQUENCY CONSTRAINTS ON ITERATES.}

$w=(2 \cdot \pi \cdot 20) / 1024$, stopband parameter $\delta_{s}=0.03$, and the time domain bound parameter $e_{i}=0.05$. The reconstruction result is comparable to the wavelet domain interpolation method described in [3]. It is possible to restore the main features of Donoho's Heavisine signal.

Convergence of the iterative algorithm can be proved using the projections onto the convex sets theorem [6], [7] because the set $C_{s}$ and sets $C_{i}$ are closed and convex sets. In Figure 3, restored signals after $1,10,20$, and 58 iteration rounds are shown.

A two-dimensional (2-D) example is provided in Figures 4, 5, and 6. The original terrain model given in Figure 4 consists of $225 \times 425$ sample points. As a first example, we assumed that one-fourth of the samples of the original signal are available in a random manner. The 2-D signal shown in Figure 5 is reconstructed using the parameters $w_{c}=\pi / 4$, $\delta_{s}=0.03$, and $e_{i}=0.01$. In the second example, we assume that one eighth of the samples of the original signal are available in a random manner. The reconstructed signal using the parameters $w_{c}=\pi / 8, \delta_{s}=0.03$, and $e_{i}=0.01$ is shown in Figure 6. Reconstruction results, which are given in Figures 5 and 6 , are low-pass filtered versions of the original 2-D signal in a dense 2-D grid.

\section{CONCLUSIONS}

It is shown that it is possible to filter a nonuniformly sampled signal by using the stopband region in the Fourier domain. The filtering concept can be easily extended to bandpass, bandstop, and high-pass filtering. Rather than defining the passband region, desired stopband regions should be defined and used in the iterative filtering process. Moreover, as a byproduct, the method can also be used for interpolating irregularly sampled data into a regularly sampled grid. In standard PapoulisGerchberg signal interpolation framework, high-frequency components are forced to take zero values. In our case, high-frequency values of the signal are allowed to take values according to stopband condition defined in (3).

\section{AUTHORS}

Kivanc Kose (kkivanc@ee.bilkent.edu.tr) is a Ph.D. student at Bilkent University. His research interests are digital image, video, and 3-D signal processing. He is a Student Member of the IEEE.

A. Enis Cetin (cetin@bilkent.edu.tr) is a professor at Bilkent University. His main research interests are multimedia signal processing and its application. $\mathrm{He}$ is a Fellow of the IEEE.

\section{REFERENCES}

[1] D. Munson, Jr. and E. Ullman, "Support-limited extrapolation of offset Fourier data," in Proc. IEEE Int. Conf. Acoustics, Speech, and Signal Processing (ICASSP'86), vol. 11, Apr. 1986, pp. 2483-2486.

[2] A. Ozbek, "Noise filtering method for seismic data," U.S. Patent 5971 095, June 23, 1998.

[3] H. Choi and R. Baraniuk, "Interpolation and denoising of nonuniformly sampled data using wavelet-domain processing," in Proc. IEEE Int. Conf. Acoustics, Speech, and Signal Processing (ICASSP), 1999, vol. 3, pp. 1645-1648.

[4] A. Ozbek, "Adaptive seismic noise and interference attenuation method," U.S. Patent 6,651,007, Nov. 18, 2003.

[5] A. Papoulis, "A new algorithm in spectral analysis and band-limited extrapolation," IEEE Trans. Circuits Syst., vol. 22, no. 9, pp. 735-742, Sept. 1975.

[6] D. C. Youla and H. Webb, "Image restoration by the method of convex projections: Part 1 theory," IEEE Trans. Med. Imag., vol. 1, no. 2, pp. 81-94, Oct. 1982.

[7] P. Combettes, "The foundations of set theoretic estimation," Proc. IEEE, vol. 81, no. 2, pp. 182-208, Feb. 1993.

[8] H. J. Trussell and D. M. Rouse, "Reducing nonzero coefficients in FIR filter design using POCS," in Proc. European Signal Processing Conf. (EUSIPCO), Sept. 2005.

[9] W. Lertniphonphun and J. McClellan, "Complex frequency response FIR filter design," in Proc. 1998 IEEE Int. Conf. Acoustics, Speech and Signal Processing, May 1998, vol. 3, pp. 1301-1304.

[10] K. Haddad, H. Stark, and N. Galatsanos, "Constrained FIR filter design by the method of vector space projections," IEEE Trans. Circuits Syst. II, vol. 47, no. 8, pp. 714-725, Aug. 2000.

[11] A. Cetin, O. Gerek, and Y. Yardimci, "Equiripple FIR filter design by the FFT algorithm," IEEE Signal Processing Mag., vol. 14, no. 2, pp. 60-64, Mar. 1997.

[12] A. E. Cetin and R. Ansari, "Convolution-based framework for signal recovery and applications," $J$. Opt. Soc. Am. A, vol. 5, no. 8, pp. 1193-1200, 1988.

[13] K. Slavakis, S. Theodoridis, and I. Yamada, "Online kernel-based classification using adaptive projection algorithms," IEEE Trans. Signal Processing, vol. 56, no. 7, pp. 2781-2796, July 2008. 